\title{
Acclimatization of Nanorobots in Medical Applications Using the Artificial Intelligence System with the Data Transfer Approach
}

\author{
Hariprasath Manoharan, ${ }^{1}$ Yuvaraja Teekaraman $\left(\mathbb{D},{ }^{2}\right.$ Ramya Kuppusamy $\mathbb{D D}^{3}$ \\ Arun Radhakrishnan (iD, ${ }^{4}$ and K. Hariprasath Venkatachalam ${ }^{5}$ \\ ${ }^{1}$ Department of Electronics and Communication Engineering, Panimalar Institute of Technology, Chennai 600 123, India \\ ${ }^{2}$ Department of Electronic and Electrical Engineering, The University of Sheffield, Sheffield S1 3JD, UK \\ ${ }^{3}$ Department of Electrical and Electronics Engineering, Sri Sairam College of Engineering, Bangalore City 562 106, India \\ ${ }^{4}$ Faculty of Electrical \& Computer Engineering, Jimma Institute of Technology, Jimma University, Ethiopia \\ ${ }^{5}$ Department of Electronics and Communication Engineering, Audisankara College of Engineering and Technology, Gudur, \\ Andhra Pradesh 524 101, India
}

Correspondence should be addressed to Yuvaraja Teekaraman; yuvarajastr@ieee.org and Arun Radhakrishnan; arun.radhakriahnan@ju.edu.et

Received 28 September 2021; Accepted 23 December 2021; Published 20 January 2022

Academic Editor: Deepak Gupta

Copyright (c) 2022 Hariprasath Manoharan et al. This is an open access article distributed under the Creative Commons Attribution License, which permits unrestricted use, distribution, and reproduction in any medium, provided the original work is properly cited.

\begin{abstract}
This article deliberates various issues that are present in the integration of nanorobots in medical applications when real-time arrangements are used. In the current generation, there is a need for a device to recognize different diseases in a fast mode of operation where data transfer should be highly accurate. Even though many devices are existing, valuable information is not provided on the detection segment that has been delivered. Therefore, the technology of nanorobots can be implemented for detecting and providing solutions to various diseases in the human body. These nanorobots operate with gesture actions and they are applied in two different surfaces. Since the robotic technology is implemented in the proposed method, it is necessary to integrate an artificial intelligence (AI) technique in amalgamation with a neural network system which feeds the input in multiple ways using hidden layers. If such arrangements are processed, then, a data transfer path will be communicated with the media access control (MAC) design where the results can be analyzed in a real-time environment using online analysis, and in turn, the outcomes are simulated using MATLAB. Subsequently, comparing the MATLAB exploration with the existing method, it can be observed that the proposed method in the application of the medical field can be prominently improved for an average of $68 \%$.
\end{abstract}

\section{Nanorobots in Medical Applications: An Indication}

In the previous generation, nearly one-third of the population in the world is facing various issues related to health care and needs early diagnosis which is necessary for proper surgical methods. In case if the diseases are not diagnosed properly at early stages, then, there is a high probability that it may lead to an individual death which should be avoided at any cause. Even though many image processing algorithms are present for early detection of nerves and materials, not even a single method is able to provide high effective results. In addition, a sensor-based technology is also introduced in medical applications which require installation of nanosensors inside the body where severe affects will raise inside the body. Therefore, to avoid such causes, a technology needs to be introduced which needs to be minuscule in size. Moreover, if nanosized particles are introduced in the field of medical applications, then, more amount of blocks will be transferred; this type of problems can be solved using single injection cases.

To solve the problem of implementation using conventional techniques in the field of medical applications, a diverse technique is needed and it is proposed that nanorobots can 
be introduced in the field of treatment for every inspection of time. It is also perceived that during such installation cases, early diagnosis can be done, thus preventing loss of lives among a group of people. Further, nanorobots differ from conventional methods in terms of ease of installation and the cost of connecting equipment will vary with respect to the designed considerations. If nanorobots are implemented, then, people can monitor their health at their remote locations which provides high modicum in fast-moving conditions. Most of the nanorobotic technology focuses on monitoring various parameters that are related to lost tissues thereby improving the productivity of medical tests and devices.

1.1. Relevant Works. In this section, the mechanisms that are related to nanorobots are discussed where all basic parametric evaluation has been perceived in a complete modus. For assessing the proposed method, a study case has been examined by using magnetic control systems where a microrobotic technology has been used for treatment of medical circumstances [1]. Since the robots are navigated in different directions, an innovative formulation for magnetic radiation has been instigated and mechanical designs with open discussion and solutions are presented. However, a magnetic control system requires a moving torque inside the robots which makes the study case to be implemented only at most necessary circumstances. When this study case has been determined, an evaluation has been made for all biomedical applications [2] in a versatile mode by linking sensing devices with nanorobots. In this case, the robots are developed using different chemical components as they vary depending on morphology and consistency. Even though high amount of consistency has been acquired, a lot of chemical parameters has been used which makes the process to be implemented in a real-life environment as it may cause hazardous actions. In real-time simulation, a three dimensional clinical database address has been protected where most of the communicating nanorobots have been integrated in basic segments [3]. This type of contrivance usually includes a satellite surveillance system that focuses on saving the lives of many people from epidemic diseases. In the absence of a satellite surveillance system, the movement of nanorobots inside blood vessels is observed [4] with an adaptive control algorithm where the destination can be reached instantaneously with high accurate measurements. But this system is not suitable under real-time applications as installation of nanorobots is a difficult task and it is not possible to keep these robots in neighboring nodes. Several basic parameters have been evaluated with rigid body trajectory paths which provide a high intelligent system for a precise design of controllers. Since the robots are constructed with supporting tissues, a high power line communication is required [5] with reliable communication rates that vary between 100 and 1000 microns. Even at high power, there is a high possibility that all constructed tissues will be unexploited if the distance of separation is maximized. Therefore, for better construction at high frequency ranges, only few neighboring nodes will be allocated that provides a good tradeoff in terms of construction and management.
Further, advanced diagnosis on medical applications has been developed that provides an exceptional capability to minimize the risk of operation where all complex tasks can be performed at a small-scale operation [6]. High technological implications can create a better opportunity for future concocts to implement nanorobots in the medical field with high image capturing proficiency. The methodology implemented in [7] suggests that if future development is needed, then, adjacent collaboration is needed with miscellaneous energy modes. However such advances are not developed and after realizing the benefits of Nano robots in medical applications such intriguing technologies are developed. If high energy is touched, then, the mode of adaptability will be increased in considered regions for all different applications with low cost of implementation. In case of gesture activities, it is required to identify a collaborative mechanism between nanorobots and different communities on medical research. Even detoxification and the approach on synthesis will be provided with rapid changes on all cutting edge technologies.

If nanorobots are applied in medical applications, the velocity of blood vessels that are flowing deep inside the body can be easily recognized [8]. The aforementioned method is demonstrated with the help of online tool analysis where all navigation information will be provided with a two-stage state estimation procedure. Conversely, the same model has been replaced by the Fourier series procedure where the movement of blood vessels is estimated with an observable behavior (i.e., if zero is present, then the system will not be observed whereas in case of 1 , the system remains in observable condition so that any flow during this period will be calculated). By extending this observable concept, the authors [9] have formulated a magnetic method which makes the nanorobots be ambitious using magnetic field configurations. In this way, interactions will happen and an actuation mechanism can be added to describe the effect of nanorobots in case of emergency situations. In addition, three steps will be followed for the design of magneticdriven robots such as understanding the design concept with different topologies, indulgent functionalities, and high automotive devices. Further, the magnetic system perception can be applied in the self-propagation mode with immediate delivery of targets [10]. This method is directly applied as an automotive technique for tumor treatment using different gesture paths.

In the current generation, the nanorobotic technology has been extended to check the DNA of individual persons and it is used for treatment of cancer with high radiation [11]. But still now, such technologies are under testing mode since the addition of targets will happen which can able to detect other diseases in the entire body. If drugs are delivered for any disease, the variety in engrossment is calculated using noninvasive techniques [12]. Since drugs are delivered at a proper time, most of the diseases can be eradicated within a short period of time, and if any side effects are found, those also will be treated immediately at a remote location in the presence of nanotechnology. Even after invention of nanorobots, AI-based technique is not integrated as devices are not precise due to the low micron size 
[13]. Due to the presence of low micron size Nanorobots are termed as controllable machines where any defected parts can be easily identified and controlled, and they can be used in all biomedical applications [14]. Furthermore, the speed of identification will be increased at low cost as compared to wearable devices. Nevertheless, the amount of fabrication needed for the emerging purposed is not mentioned due to negative impulse response variants [15]. The aforementioned works are developed with respect to nanotechnology fields and all interventions are tested with some limitations.

1.2. Research Gap and Motivation. Most of the existing works focuses only on the implementation of conventional devices that will not help the people to monitor themselves at their remote locations. However, if any sudden discomfort occurs, people can visit nearby infirmaries and they can check their vigor after waiting for a long period of time. Even the same method exists in all parts of the world, and societies still believe the same way which is organized like the older day periods. But on the other side, a number of technologies have been developed with many devices which are very minute in sizes, and the usage of such intellectual devices is lesser.

Therefore, this research article identifies the gap that is not present in an existing conventional device and discovers a new way to nanorobotic solution for applying in medical applications. However, at initial stage of installation, some motions are difficult to detect but after equivalent training models, the proposed work on nanorobots performs much better than existing case studies $[1,2]$. The foremost impetus of the projected work is to implement such artificial intelligent nanorobots at low cost for all individuals in the society to detect the presence of diseases at the early stage.

1.3. Objectives. The major objective of the proposed research is to introduce a nanorobot in the field of medical diagnosis by combining multisensing models for early diagnosis of both tissues and muscles by solving the ensuing basic robotic parameters.

(i) To measure the motion of a nanorobot using corresponding weights for external control process

(ii) To detect the motion error for allocating sufficient resources at corresponding intervals

(iii) To maximize the transmission capability of nanorobots for transferring the information at maximum possible routed ways

For implementing the abovementioned objectives, an AI technique has been introduced where training data has been incorporated at starting stages with a set of predetermined values. Once the values are obtained, a neural network has been introduced with different weightage process that detects the ailment of different individuals.

\section{Problem Formulation}

In this section, a new flanged mathematical formulation that is used for calculating the exact scenarios of nanorobots is provided with high benefits of integration at corresponding segments. In this case, as a first segment, transmission of signals is calculated from the reference path using different weights as follows

$$
R_{i}=\sum_{i=1}^{n} a_{n} x_{n}
$$

where $a_{n}$ and $x_{n}$ denote the corresponding nanorobots that are installed with larger encumbrances.

In the proposed method, nanorobots are designed in such a way where the moment is restricted to either a flat or perpendicular level. For both directions, discrete equations should be framed and it can be represented in terms of error as

$$
e_{i}=\left\{\begin{array}{l}
x_{t}-x_{m} \text { for flat, } \\
y_{t}-y_{m} \text { for perpendicular, }
\end{array}\right.
$$

where $x_{t}$ and $x_{m}$ indicate the motion of nanorobots for a flat surface in both curved and gesture paths, respectively. $y_{t}$ and $y_{m}$ indicate the motion of nanorobots for a perpendicular surface in both curved and gesture paths, respectively.

Equation (2) indicates that there will be some system error with respect to different motions in nanorobots and they can be installed in both surfaces in a precise method. This equation is framed in order to reduce the error during arrival of different motion paths. Therefore, oscillation of nanorobots should be calculated using negative force values with integral values using equation (3).

$$
F_{i}=\int_{S e i}^{n} \rho_{i} \varphi_{i} d \varphi
$$

where $\rho_{i}$ denotes the density of flux over the given surface area. $\varphi_{i}$ represents the area covered in all directions with finite integral values.

Since in equation (3), the flux density is represented, a power line communication for transmission of signals should be inserted in the body surface of nanorobots. Therefore, the power line communication at corresponding time periods can be expressed using equation (4) as follows

$$
P_{i}=\frac{1}{2} \sum_{i=1}^{n} \aleph\left(F_{i} * \vartheta_{i}\right)
$$

where $\aleph\left(F_{i} * \vartheta_{i}\right)$ represents the average power with respect to time that entails force and velocity parameters.

Equation (4) indicates the transmission power capability of only standard nanorobots. But the same equation will be varied for spherical nanorobots, and the design constellation will also differ for such robots that are not examined in the proposed method. If the power supplied to nanorobots are higher, then, constraint on heat will become complex, and to avoid such complexities, equation (5) is framed with spherical power density as

$$
S P_{i}=\frac{P_{i}}{\text { surface area of sphere }},
$$


where the surface area of sphere $=(4 / 3) \pi r^{3}$ should be calculated for avoiding of the complex condition related to temperature relationships.

Equation (5) can also be expanded using the thermal relationship on spherical conditions that satisfies the temperature constraint using the dispersion equation as follows

$$
T_{i}=\frac{\circ F_{i}}{\circ \mathrm{C}_{i}} \nabla_{i}^{2}
$$

where $\circ F_{i}$ and $\circ C_{i}$ denote the outer tissue that contains thermal and heat capacity of nanorobots, respectively.

Equation (6) represents that maximum endurable limits should be established before insertion of tissues inside the nanorobots. In addition, a steady-state temperature should be established for any boundary that exceeds within a certain limit as warning indications can be given at a short period of time. Therefore, the susceptibility of nanorobots can be given using equation (7) as

$$
\omega(i)=\sum_{i=1}^{n} \frac{M_{i}}{\epsilon_{i}}
$$

where $M_{i}$ and $\epsilon_{i}$ denote the corresponding applied mass and external electric field, respectively, in order to get physical representation for the type of magnetic penetrability in robots.

Equation (7) indicates that the supplied external electric field should be minimized; therefore, aggregate vulnerability characteristics of nanorobots can be minimized. In addition, another important parameter is the cost of installing tissue around the robots which gives a complete round off values using equation (8).

$$
C_{i}=\sum_{i=1}^{n} \partial_{i} \gamma_{i}
$$

where $\partial_{i}$ and $\gamma_{i}$ denote the tissues and weight, respectively, of tissues that are covered at the outer end of robots.

It is factual that the cost of implementation depends only on tissue values, and therefore, for each flat of the perpendicular surface, the measurement should be precise which in turn leads to minimization of the cost.

\section{Optimization Algorithm}

Since nanorobots are designed with tissues, it is necessary to implement a set of rules for passing the information from the transmitting to the receiving station. In this case, an infrastructureless network has been designed using ad hoc wireless network since the robots are present at different nodes that move with respect to time. In addition, since wireless networks are used as communication module, it is necessary to implement a media access control (MAC) protocol that uses a common clock for synchronizing the packets with respect to each other. Moreover, the designed network uses the distributed mode of operation in the absence of concealed and exposed stations. The parameters for designing the MAC protocol for the operation of the nanorobot can be expressed in mathematical form as

$$
K_{1}=\sum_{i=1}^{n} \frac{\theta_{1}}{2} * \beta_{i}+T R_{x i}
$$

where $\theta_{1}$ denotes the single time period frame. $\beta_{i}$ represents the duration of the preamble. $T R_{x i}$ denotes the transmission and reception power of MAC protocols.

If equation (9) is designed correctly, then, even at low bandwidth and power, the robots is able to transfer information to all corresponding neighboring nodes. Further, resources will also be allocated for avoiding the delay in the entire network which can be expressed as

$$
d_{i}=\sum_{i=1}^{n} \frac{\theta_{i}}{2}\left(t_{d}+t_{e a}+t_{d a}\right),
$$

where $t_{\mathrm{d}}, t_{\mathrm{ea}}$, and $t_{\mathrm{da}}$ denote the time taken for data transfer, early acknowledgment, and data transfer acknowledgement at different phases.

Since the robotic technology is used, an artificial intelligence $(\mathrm{AI})$ technique $[16,17]$ is integrated for further process verification using multihop networks. Therefore, a multilayer feed forward mechanism is implemented where it is easier to implement an omnidirectional antenna for data transfer in one direction. When such multiple layers are designed, then, errors in data should be minimized. This can be integrated with equation (2) as follows

$$
E_{i}=\sum_{i=1}^{n} \frac{1}{2}\left(O_{i}-\widehat{O_{i}}\right),
$$

where $O_{i}$ and $\widehat{O_{i}}$ denote the observed output and predicted output values, respectively, for a single time period.

If errors are minimized, then, binary classification can be applied for all packets that are transmitted to the destination. The binary classification can be modeled using a binary number as expressed in equation (12).

$$
O_{i}=\left\{\begin{array}{l}
1 \text { if } \text { error }=0 \\
0 \text { if } \text { error }=1
\end{array}\right.
$$

The predicted output is related to the activation function of different neurons which can be expressed in mathematical form using equation (13).

$$
\widehat{O_{i}}=\frac{E_{T}}{\sum_{i=1}^{n} E_{i}},
$$

where $E_{T}$ denotes the total error after combining equation (2) and (11).

The major advantage of implementing the multilayer feed forward mechanism in AI is that it can be integrated with many problem domains in different areas as their topology is very easy to implement. Moreover, a universal 


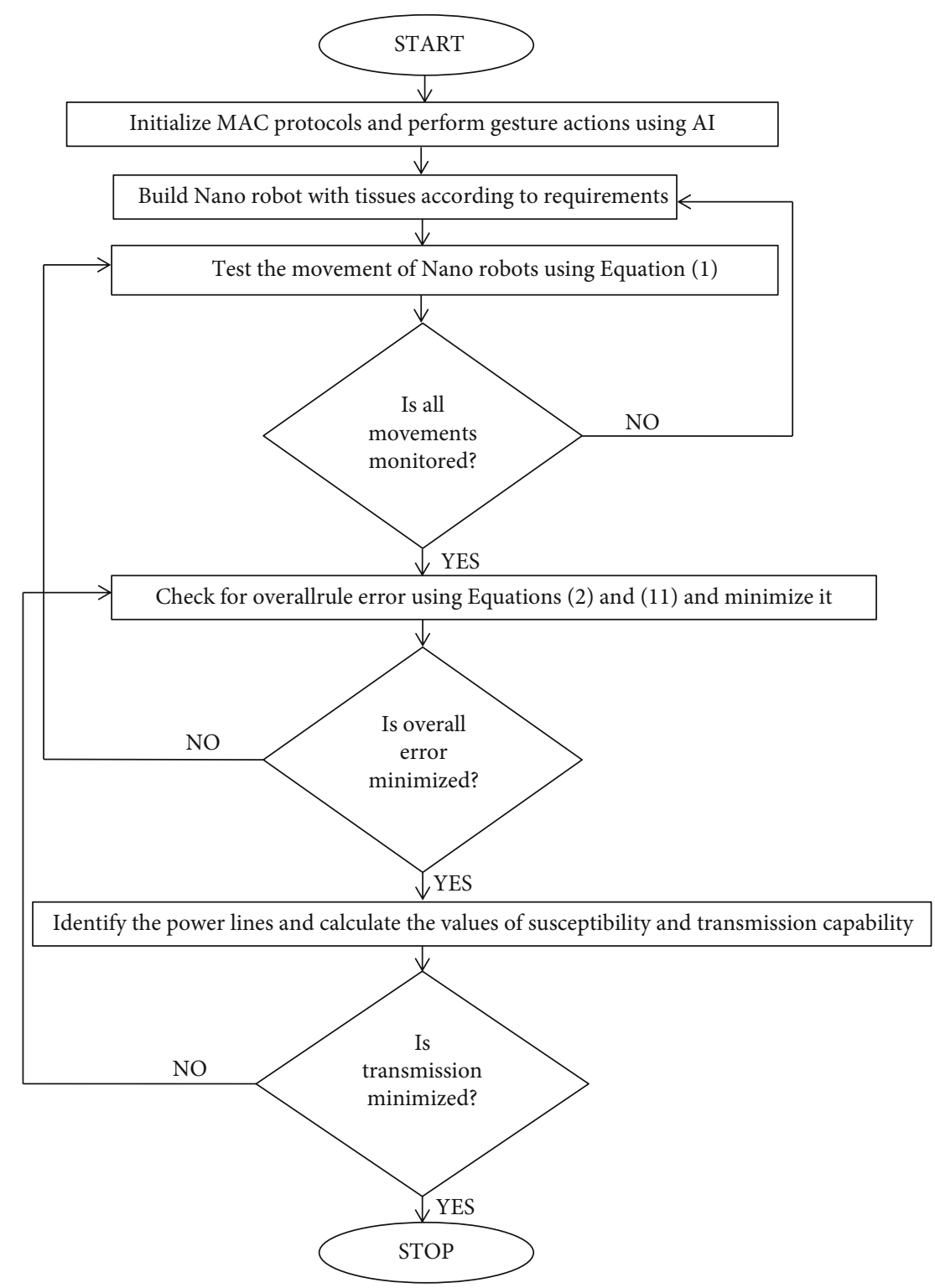

FIGURE 1: Process of assimilating nanorobots for medical applications using AI.

approximation problem can be solved using this type of algorithm where all nonlinear dynamic systems can be activated using composite materials. In the multilayer perceptron mechanism, the images can be easily classified by different robots since the input variables are not limited in their topology even though they are present in complex networks. The cost of implementing such networks can be integrated with equation (8) as

$$
C_{i}\left(w_{i}, b_{i}\right)=\frac{1}{2 \nabla}(|a|)
$$

where $w_{i}$ and $b_{i}$ indicate the corresponding weights and input biases of feed forward networks, respectively. $|a|$ denotes the output vector from multiple layers.

Equation (14) denotes that cost function of the integrated algorithm is calculated with respect to weights and biases that are supplied from three different layers such as input, hidden, and output layers.

Also, the aforementioned equation represents the weighted least square mechanism that is universally used for all neural network algorithms for nanorobotic technology. Figure 1 shows the step-by-step process of designing nanorobots for medical applications using AI.

\section{Results and Discussion}

In this section, real-time analysis on the performance of microrobots for medical applications has been verified by measuring different parameters. The purpose of measuring different parameters will assure better quality of service, and in turn, these values can be observed using an online monitoring system. In addition, for all these scenarios, a set of rules have been defined that provides support for high-reliability and scalability measures. All the observed 
parametric values are considered as different scenarios as follows-scenario 1: motion of the nanorobot, scenario 2: motion error, scenario 3: transmission capability, scenario 4: susceptibility, and scenario 5: cost of implementation.

To provide better understanding, all scenarios are plotted in MATLAB where three dimensional images are strategized. Also, an additional assumption has been considered for achieving a high-performance operation where the size of each robot should be much higher. This assumption is made in order to provide a self-organizing capability since the nodes are in moving position with respect to each other. By following the assumption for five different scenarios, descriptions on plots have been provided as follows.

4.1. Scenario 1. In this scenario, the motion of nanorobots with respect to different weights has been observed. The major reason for elucidating this scenario is that an artificial force will be present as an external controller where the user is not able to control the motion of robots if they are going in the wrong direction. Therefore, it is necessary to use an omnidirectional antenna for calculating the motion of nanorobots as energy is spread equally in all the directions even in the presence of an external controller. In the first step, an obstacle-free path should be identified under realtime applications, and by using the desired arrangement, all the shortest paths should be identified within a short period of time. Therefore, the motion of nanorobots is difficult to design, and in the proposed method, a new topology has been used and the corresponding motion has been identified and plotted using Figure 2.

In Figure 2, it can be observed that the motion of nanorobots is observed for different meters according to the corresponding weights in kilograms. Also, from the contour plot, a distance in hundred meters can be extended as a maximum time period for wave motion. Therefore, from the observed results for different weights, the proposed method proves to be much efficient than the existing method [3]. For example, if the weight of the nanorobot is $30000 \mathrm{~kg}$, then, the motion of the nanorobot is observed as 1691 meters for the proposed method whereas with same amount of weight, the existing method has limited the motion of nanorobots to 1004 meters which is much less than the proposed method.

4.2. Scenario 2. Once the motion of nanorobots is maximized, then, in the next case, error values in terms of micrometers should be observed under two different surfaces such as flat and perpendicular. Error values will be calculated from equation (2) using two different paths such as gesture and curved. In addition, the overall error values are calculated from equation (11) where predicted output values will be observed. In the proposed method, an infrastructureless network has been designed where error values should be minimized (i.e., gesture paths should be chosen correctly in accordance with resources that are allocated at the first stage). The observed error values are plotted in Figure 3 under offline analysis.

In Figure 3, error values in terms of micrometers are plotted at different time periods ranging from 5 seconds to 50 seconds. For different time periods, the error values

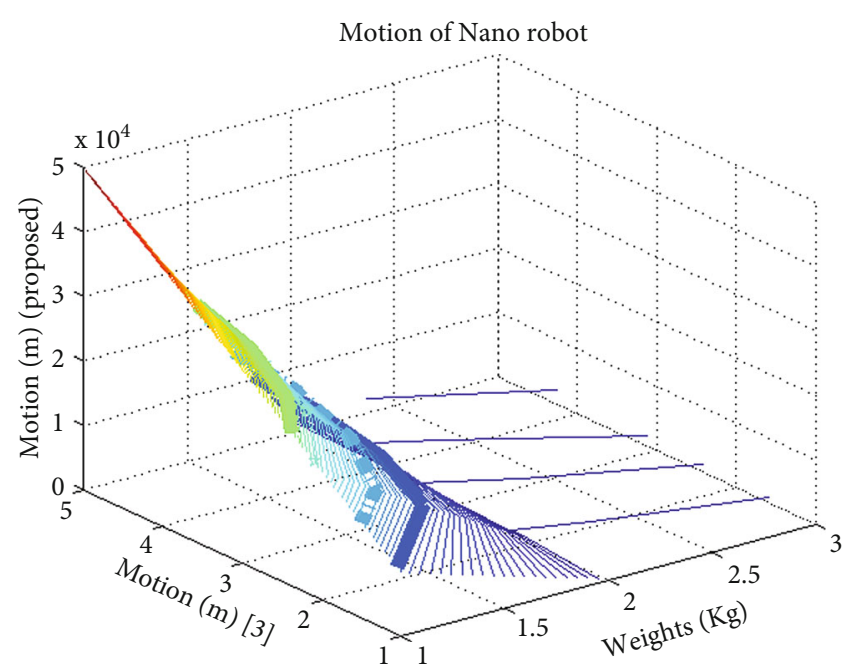

FIGURE 2: Motion of nanorobots.

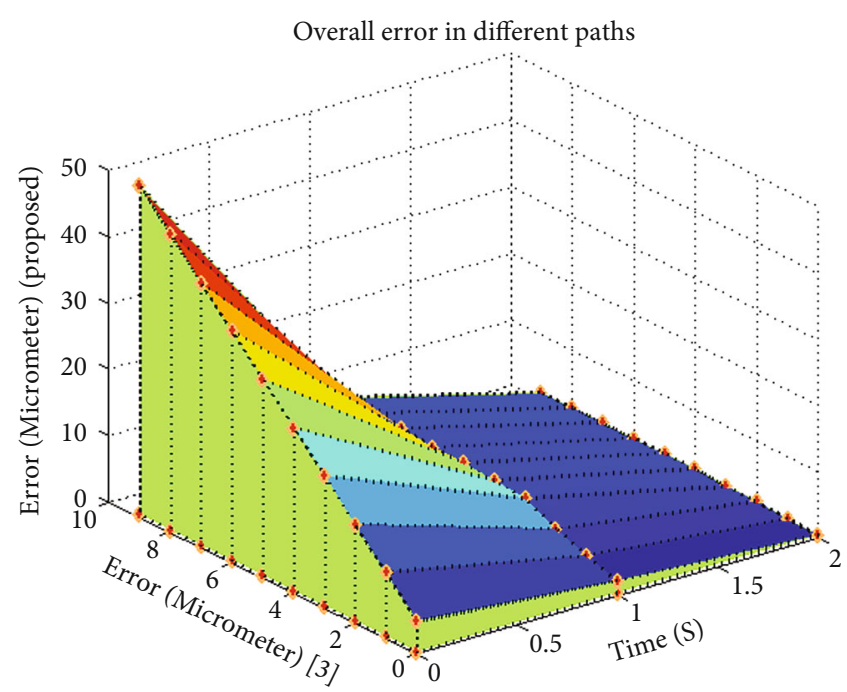

Figure 3: Motion errors of nanorobots.

should decrease or it should remain at a constant rate. If the aforementioned condition is satisfied, then, it is much easier to observe the motion of robots and even it can be controlled at a corresponding time period. The mesh plot indicates that error values for the proposed method are much lesser than the existing method [3]. For example, if the time period is 30 seconds, then, the overall error rate can be observed as 8.6 micrometers for the existing method whereas for the proposed method, the error rate remains constant after 30 seconds until the maximum time period is accomplished.

4.3. Scenario 3. In this scenario, the minimum transmission capability of the designed nanorobots in medical applications has been observed. Transmission capability defines the rate of how deep an image can be captured and reported to the neighboring nodes under mobility. If the transmission capability of nanorobots is lesser, then, a clear image can be captured, and if any problem occurs inside the human body, 


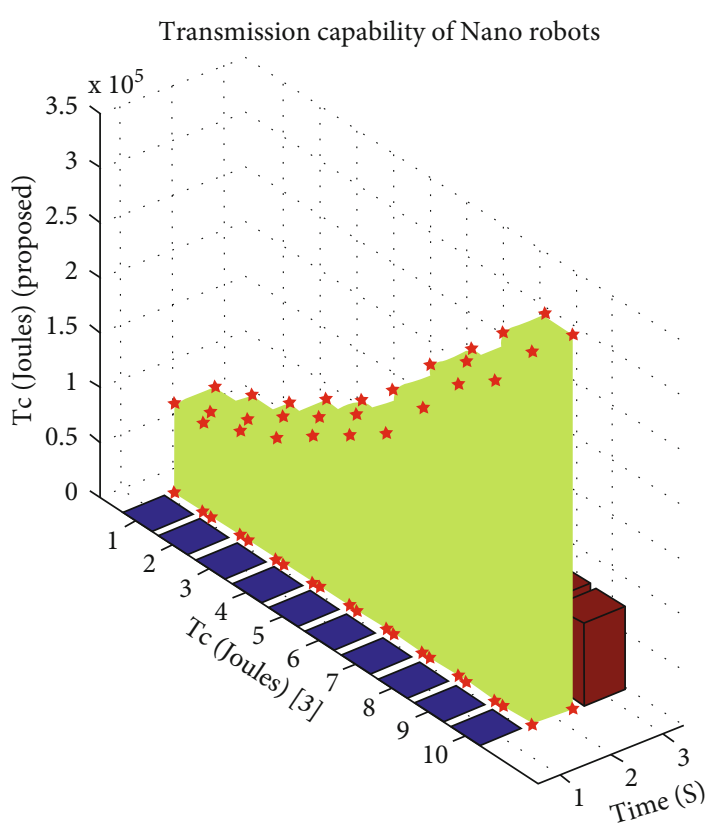

FIgUre 4: Capability of transmission.

it will be detected within a short period of time. But if the designed robots are having high transmission capability, identification of diseases will take a long period of time which in turn makes the designed methodology inefficient. After observing the minimum capability rate, the results are simulated in offline analysis and are shown in Figure 4.

In the three-dimensional bar graph in Figure 4, it is implicit that the transmission capability of the proposed method using nanorobots is much lower which is expressed in Joules. For a clear view, the time period is considered from 5 to 50 seconds where within the corresponding time period, transmission capability remains constant for the proposed method from 45 seconds. Moreover, if the time period is 30 seconds, then, the transmission capability is observed to be 178000 Joules for the existing method, but for same time period, the proposed method provides low capability rate which is equal to 68000 Joules. This proves that even the capability rate can be minimized or it can be kept constant for the designed nanorobots.

4.4. Scenario 4. In this scenario, the vulnerability rate of response received from nanorobots has been observed at a corresponding distance. If the distance is increased or decreased, the vulnerability rate should be either maximized or minimized depending on gesture actions. But in the design of nanorobots, the vulnerability rate should always be minimized since the nanorobots are designed with highend tissues where gesture actions will always be accurate. Therefore, for different gesture actions with high distance, the rate of vulnerability is observed and plotted in Figure 5.

Figure 5 is plotted by considering 31 different data sets which varies with respect to distance and time periods. It can be observed in Figure 5 that as the distance of separation increases, the susceptibility rate which is measured in micrometer per kilogram is increased, and for the proposed

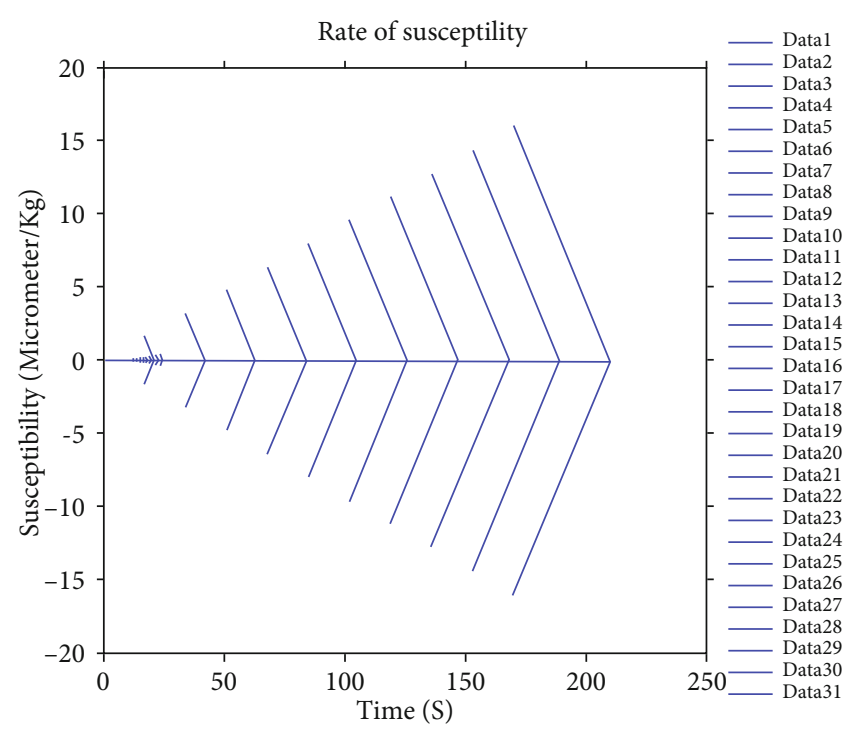

Figure 5: Vulnerability rate for 31 datasets.

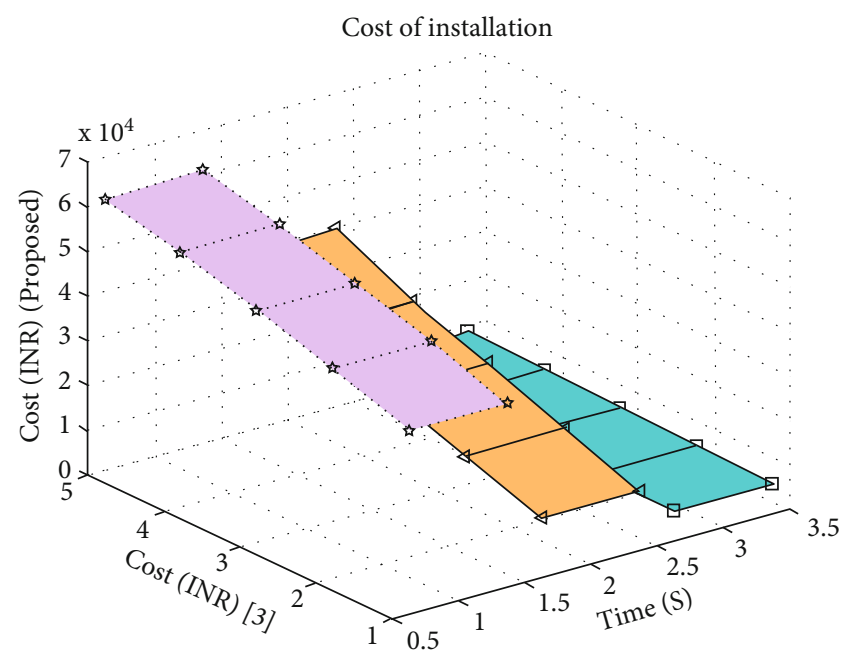

Figure 6: Cost of implementation.

method, after a distance of 80 meters, the vulnerability rate is observed to be constant even though the data is varied to maximum extent. But for the existing method [3], even though data varies, the susceptibility rate increases to maximum extent which does not guarantee proper operation rate even for 200 meters.

4.5. Scenario 5. The aforementioned scenario focuses on hypothetical segments that are necessary for the operation of nanorobots. But in this scenario, the cost of implementing nanorobots according to the designed parameters is calculated. Since in the proposed method, nanorobots are designed for medical applications, only few parameters are considered, and as a result, the cost of implementation will be lesser than those of standard methods. The cost of implementation is measured in Indian Rupees, and the same is plotted using the ribbon plot in Figure 6 . 
In Figure 6, it can be observed that cost of installation is much lesser for the proposed method and is calculated using equations (8) and (14). It is apparent that as time varies, the cost of implementation becomes lesser, and depending on the transmission capability between 41000 and 61000, the cost will be varying and maximum Rs. 8500 can be consumed for building such nanorobots. This proves that the proposed method is highly valuable during real-time implementation of different networks under the presence of MAC protocol.

\section{Conclusions}

The proposed work uses a new flanged technology on the nanorobotic mechanism in medical applications where its performance is tested under basic parametric values. In case of pandemic circumstances, a real-time application on medical diagnosis is always necessary for saving the life of humans. Even if diseases cannot be cured, at least, it should be identified and spread rate should be controlled at a higher proportion. With such high technologies on nanorobots, medical applications can be developed and any disease can be identified within a short period of time. Moreover, many developed methods provide high advantage on real-time applications but a highly efficient algorithm integration is not provided in any advanced technologies. But the proposed method integrates an AI technique with the MAC protocol for designing a set of rules with the data transfer stage. If these technologies are integrated with each other, then, automatic monitoring of robots can be created at low cost with different tissues, and in real time, this technology can be installed using different gesture actions. The proposed technology is tested under five different scenarios, and overall parametric values are also calculated where the proposed method proves to be much efficient even if it is installed in real time.

\section{Data Availability}

The data used to support the findings of this study are available from the corresponding author upon request.

\section{Conflicts of Interest}

The authors declare that they have no conflicts of interest.

\section{References}

[1] Y. Shao, A. Fahmy, M. Li, C. Li, W. Zhao, and J. Sienz, "Study on magnetic control systems of micro-robots," Frontiers in Neuroscience, vol. 15, pp. 1-15, 2021.

[2] L. Wang, Z. Meng, Y. Chen, and Y. Zheng, "Engineering magnetic micro/nanorobots for versatile biomedical applications," Advanced Intelligent Systems, vol. 3, no. 7, article 2000267, 2021.

[3] A. Cavalcanti, B. Shirinzadeh, M. Zhang, and L. C. Kretly, "Nanorobot hardware architecture for medical defense," Sensors, vol. 8, no. 5, pp. 2932-2958, 2008.

[4] A. Farahani and A. Farahani, "An adaptive controller for motion control of nanorobots inside human blood vessels,"
Bioscience Biotechnology Research Communications, vol. 9, no. 3, pp. 546-552, 2016.

[5] T. Hogg and R. A. Freitas, "Acoustic communication for medical nanorobots," Nano Communication Networks, vol. 3, no. 2, pp. 83-102, 2012.

[6] F. Soto and R. Chrostowski, "Frontiers of medical micro/ nanorobotics: in vivo applications and commercialization perspectives toward clinical uses," Frontiers in Bioengineering and Biotechnology, vol. 6, pp. 1-12, 2018.

[7] C. Yamamuro, J.-K. Zhu, and Z. Yang, “乳鼠心肌提取 HHS public access," Physiology \& Behavior, vol. 176, no. 12, pp. 139-148, 2017.

[8] M. Fruchard, L. Arcese, E. Courtial et al., Estimation of the Blood Velocity for Nanorobotics to Cite This Version : HAL Id : Hal-01071275 Estimation of the Blood Velocity for Nanorobotics, 2014.

[9] H. Zhou, C. C. Mayorga-Martinez, S. Pané, L. Zhang, and M. Pumera, "Magnetically driven micro and nanorobots," Chemical Reviews, vol. 121, no. 8, pp. 4999-5041, 2021.

[10] S. S. Andhari, R. D. Wavhale, K. D. Dhobale et al., "Self-propelling targeted magneto-nanobots for deep tumor penetration and PH-responsive intracellular drug delivery," Scientific Reports, vol. 10, no. 1, pp. 1-16, 2020.

[11] R. Devasena Umai, P. Brindha Devi, and R. Thiruchelvi, "A review on Dna nanobots - a new technique for cancer treatment," Asian Journal of Pharmaceutical and Clinical Research, vol. 11, no. 6, pp. 61-64, 2018.

[12] P. Khulbe, "Nanorobots: a review," International Journal of Pharmateutical Sciences and Research, vol. 5, no. 6, pp. 21642173, 2014.

[13] A. Kumar and S. Roy, Amandeep Kumar, Ramya CB, Surashree Roy 8, vol. 3, no. 2, pp. 1725-1729, 2014.

[14] R. B. Durairaj, J. Shanker, and M. Sivasankar, "Nano robots in bio medical application," in IEEE-International Conference on Advances in Engineering, Science and Management, ICAESM2012, Nagapattinam, India, 2012.

[15] F. Novotny, H. Wang, and M. Pumera, "Nanorobots: machines squeezed between molecular motors and micromotors," Chem, vol. 6, no. 4, pp. 867-884, 2020.

[16] G. F. Luger, "Artificial intelligence: structures and strategies for complex problem solving," Zywienie Czlowieka I Metabolizm, vol. 5th, 2005http://www.amazon.com/dp/0321545893.

[17] G. Lample, Deep Learning for Symbolic Mathematics, pp. 1-24, International Conference on Learning Representations (ICLR), 2020. 